\title{
Novel Design of Photonic Digital Sensors for Distinguishing Uniformly-Distributed Refractive Indices of Fluids
}

\author{
R.-C. Lu \\ Department of Electronic Engineering, National I-Lan University, I-Lan City, I-Lan County, Taiwan, ROC, E- \\ mail: rclu@ems.niu.edu.tw \\ K.-Y. Lee \\ Department of Electrical Engineering, Chinese Culture University, Taipei, Taiwan, ROC, E-mail: \\ kyleentu@ms52.hinet.net
}

\begin{abstract}
The authors propose a novel structure of $k$-level ( $k$ is an arbitrary integer) photonic digital sensor based on directional couplers with specific structural parameters. Its function is similar to an analog-to-digital converter (ADC) in electronic system, and capable of distinguishing $2^{k+1}-1$ discrete and uniformly-distributed refractive indices of the fluidic samples. In this paper, the principle of designing the photonic digital sensor is described, and the beam propagation method (BPM) simulation results are presented for demonstrating the validity of our design.
\end{abstract}

Index Terms - Photonic digital sensor, beam propagation method (BPM).

\section{INTRODUCTION}

Photonic digital components and systems have been studied for a long time [1-3]. These components/systems are capable of quickly-processing the binary data or other digital signals with more complicated formats. Especially in biochemical tests, high sensitivity and rapid detecting should be fulfilled. For detecting different biochemical substances, we can distinguish them by measuring their respective refractive index. For example, Takeo and Hattori proposed an optical fibler sensor for measuring a liquid refractive index [4]. The optical power leaking from the fiber depends on the refractive index of the liquid, and a digital voltmeter is required to read the output. Moreover, Zhu proposed a high-sensitivity sensor based on various optical integrated devices [5]. The refractive index change could be determined by measuring wavelength shift. Up to now, more and more optical fiber/waveguide devices have been utilized for detecting biochemical samples [6-11]. Among these devices, the structure comprising optical directional couplers is very attractive because it is easy to fabricate compact, well-performing and low-cost sensor to detect biochemical substances. However, conventional analog sensors need precise measurements of the output optical intensities for detecting refractive indices. The total procedures of calibration and measurement may consume much time. For these reasons, the authors decide to start researching and designing the photonic digital sensors based on optical directional couplers with specific lengths for quickly testing biochemical fluidic samples. And then we have designed a novel $k$-level photonic digital sensor with $2^{k}(\forall k \in N)$ output ports. The $k$-level photonic digital sensor contains $2^{j-1}$ directional couplers in the $j_{\text {th }}$ level $(1 \leq j \leq k)$ and the total Brazilian Microwave and Optoelectronics Society-SBMO received 01 Nov 2017; for review 09 Nov 2017; accepted 03 March 2018 
number of the directional couplers within the sensor is $2^{k}-1$. Its performance is very similar to an analog-to-digital converter (ADC). Without necessity of precise measurements of the light intensities at output ports of the sensor, we just need to observe and judge which one output port emits the major optical power, and then the corresponding refractive index of the fluidic sample is obtained. The output pattern that there is only one bright output port but the others are in dark is classified as the first type. The $k$-level photonic device is capable of distinguishing $2^{k}$ discrete and uniformlydistributed refractive indices of tested fluidic samples by observing the first type of output patterns. For the second type of output patterns, it is just necessary to observe and judge which two output ports are bright and the others are in dark. And then the extra $\left(2^{k}-1\right)$ species of fluidic samples with discrete and uniformly-distributed refractive indices can be determined. Therefore, the total number of distinguishable species of fluidic samples with discrete refractive indices is $2^{k+1}-1$. Moreover, much time of precisely-measuring the light intensities at output ports and computing their mutual ratios can be saved. In this work, the authors present some simulation results to demonstrate the validity of our design.

\section{DESIGN PRINCIPLES OF K-LEVEL PHOTONIC DIGITAL SENSORS FOR TESTING FLUIDIC SAMPLES}

First of all, we select the simplest case of $k=1$ as an example to design a one-level photonic digital sensor of distinguishing fluidic samples with $3\left(=2^{k+1}-1\right.$ for $\left.k=1\right)$ discrete and uniformly-distributed refractive indices, and explain its operation for easier comprehension. The one-level photonic digital sensor contains only one $\left(=2^{j-1}=2^{1-1}=1\right.$ for $\left.j=1\right)$ directional coupler with some specification in structural parameters as the following description. Its top view and the 3D structures are shown in Figure 1(a). The width and height of the waveguide are $w$ and $t$, respectively. And the distance between the adjacent waveguides within each directional coupler is $d$. The refractive indices of the substrate, waveguide core, and the upper cladding are denoted by $n_{\mathrm{s}}, n_{\mathrm{g}}$, and $n_{\mathrm{c}}$, respectively. For easily avoiding mutual interference in the output ports, the two closely-coupled adjacent waveguides have extra $S$-bent sections to make the output ports farther away from each other. The lateral and the longitudinal offsets of the $S$-bent section can be respectively determined by numerical simulation such that the relative optical power loss in the bending section is within a very small tolerance. Denote $L_{1,1}$ as the length of the closely-coupled section of the directional coupler. Let $B_{\mathrm{z}, 1}$ and $B_{\mathrm{x}, 1}$ separately denote the lateral and the longitudinal offsets of the each $S$-bend of the directional coupler. The distance between two adjacent output ports of the photonic digital sensor is denoted by $G$. A droplet of undetermined fluid was deposited on the surface of the photonic digital sensor, and then the fluid spreads and covers almost the entire surface as shown in Figure 1(b). Before depositing the fluid droplet, the cladding between/on the top of the ridge waveguide cores is air and $n_{\mathrm{c}}=1$. After spreading the liquid/solution on the surface of the device, the value of $n_{\mathrm{c}}$ is equal to the refractive index of the droplet of the fluidic sample. Assume the lowest and the highest values of refractive indices of all the 
tested fluidic samples are $n_{\mathrm{cl}}$ and $n_{\mathrm{ch}}$, respectively.
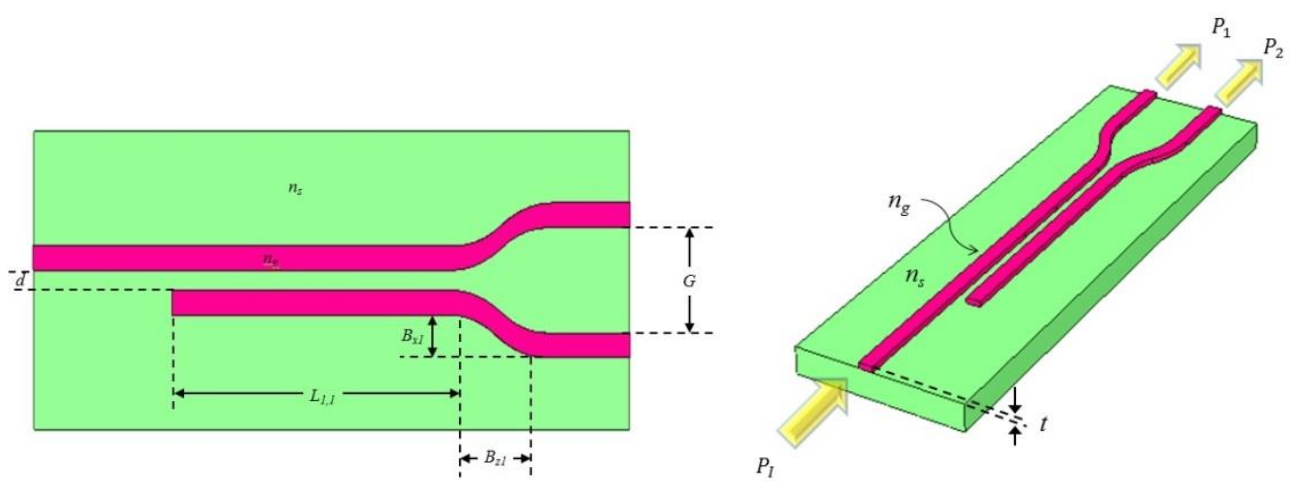

(a)

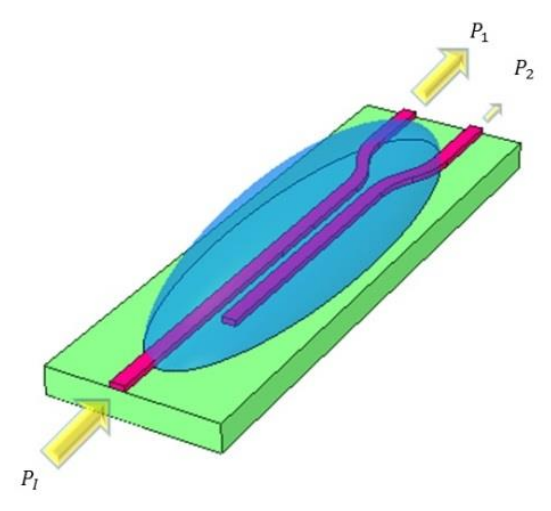

(b)

Fig. 1 (a) The top view of single-level photonic digital sensor and its 3D structure. (b) A droplet of unknown biochemical liquid/solution is deposited on the surface of the sensor. And then it spreads and covers the waveguides in the sensor, and becomes the new cladding material of the ridge waveguides. If we inject the laser beam into the input waveguide, only one output port, corresponding to a certain refractive index of the fluidic sample, is capable of emitting the majority of optical power.

For a general $k$-level photonic digital sensor, we define

$$
\begin{aligned}
& \Delta \mathrm{n}=\frac{n_{c h}-n_{c l}}{2^{k}-1} \\
& n_{i}=n_{c l}+i \Delta n
\end{aligned}
$$

and

$$
n_{l+0.5}=n_{c l}+(l+0.5) \Delta n
$$

for $0 \leq i \leq 2^{k}-1$ and $0 \leq l \leq 2^{k}-2$. And two special cases of (1b) are $n_{0}=n_{\mathrm{cl}}$ and $n_{m-1}=n_{\mathrm{cl}}+\left(2^{k}-1\right) \Delta n=n_{\mathrm{ch}}$. It is 
known that the refractive index $n_{\mathrm{c}}$ of the upper cladding (fluidic sample) increases towards that of the core region $n_{\mathrm{g}}$, the coupling length between two adjacent parallel waveguide decreases because the confinement of the optical power to the both core regions becomes worse such that power coupling between the two waveguides occurs more easily [12]. Therefore we have $L_{\mathrm{cl}}>L_{\mathrm{ch}}$, where $L_{\mathrm{cl}}$ and $L_{\mathrm{ch}}$ are the coupling lengths corresponding to $n_{\mathrm{cl}}$ and $n_{\mathrm{ch}}$, respectively. We define

$$
\Delta \mathrm{L}=\frac{L_{c l}-L_{c h}}{2^{k}-1}
$$

In case of small $\Delta n$, the coupling length $L_{\mathrm{c}, \mathrm{i}}$ corresponding to $n_{\mathrm{i}}\left(0 \leq i \leq 2^{k}-1\right)$ and $L_{\mathrm{c}, l+0.5}$ corresponding to $n_{l+0.5}\left(0 \leq l \leq 2^{k}-2\right)$ can be approximately expressed as

$$
\begin{gathered}
L_{c i i}=L_{c, 0}-i \Delta L \\
L_{c, l+0.5}=L_{c, 0}-(l+0.5) \Delta L
\end{gathered}
$$

where $L_{\mathrm{c}, 0}\left(=L_{\mathrm{cl}}\right)$ is the coupling length corresponding to $n_{0}\left(=n_{\mathrm{cl}}\right)$ and $\Delta L$ is much smaller than $L_{\mathrm{c}, 0}$. For the previously-described single-level photonic digital sensor $(k=1)$ shown in Figures 1(a)-(c), utilizing (2) and (3a), the length $L_{1,1}$ is selected as follows

$$
L_{1,1}=\operatorname{ROUND}\left(\frac{L_{c, 1}}{\Delta L}\right) L_{c, 0} \approx\left[\operatorname{ROUND}\left(\frac{L_{c, 1,1}}{\Delta L}\right)+1\right] L_{c, 1}
$$

where $\operatorname{ROUND}(\cdot)$ is the symbol to represent the rounding of the argument to the nearest integer. And the following approximation has been utilized in (4): $\operatorname{ROUND}\left(L_{\mathrm{c}, 2} / \Delta L\right) \approx L_{\mathrm{c}, 2} / \Delta L$ and $L_{\mathrm{c}, 0} / L_{\mathrm{c}, 1} \approx 1$ in case of $\Delta L<<L_{\mathrm{c}, 0}$. As for the case of $k=1$, the numerator of the argument in $\operatorname{ROUND}(\cdot)$ of (4) is $L_{\mathrm{c}, 1}$, which is the shortest one among the two coupling lengths: $\left(L_{\mathrm{c}, 0}, L_{\mathrm{c}, 1}\right)$.

The basic operation theory of the above single-level photonic digital sensor is described as follows. Firstly, assume $\operatorname{ROUND}\left(L_{\mathrm{c}, 2} / \Delta L\right)$ is even, it means that $L_{1,1}$ in (4) is an even multiple of $L_{\mathrm{c}, 0}$ but approximately an odd multiple of $L_{\mathrm{c}, 1}$. If the refractive index of the fluidic droplet is $n_{0}\left(=n_{\mathrm{cl}}\right)$, the majority of the incident light will arrive at the upper output port in the top view of the directional coupler in Fig.1(a). Therefore, the upper output port emits strong light but the lower output port is almost in dark. In case the refractive index of the fluid droplet is $n_{1}\left(=n_{\mathrm{cl}}+\Delta n\right)$, the major portion of the optical power will be coupled to the lower output port in the top view of the directional coupler. And thus the upper output port is almost in dark but the lower output port radiates bright light. Conversely, if $\operatorname{ROUND}\left(L_{\mathrm{c}, 2} / \Delta L\right)$ is odd, all the above output results are exchanged. For the cases those only one Brazilian Microwave and Optoelectronics Society-SBMO received 01 Nov 2017; for review 09 Nov 2017 ; accepted 03 March 2018 
output port of the sensor can emit strong light but the other one is almost in dark, they belong to the first type of output patterns. Therefore, a single-level photonic digital sensor based on a specificlength directional coupler can classify 2 kinds of fluid samples with different the refractive indices, $n_{0}$ and $n_{1}$, by observing the first type of output patterns. On the other hand, if the refractive index of the fluid droplet is $n_{0.5}\left(=n_{\mathrm{cl}}+0.5 \cdot \Delta n\right), L_{1,1}=\left(L_{\mathrm{c}, 0}+L_{\mathrm{c}, 1}\right) / 2$ by (3a) and (3b), so the two output ports radiate the same magnitude of lights. It is defined as the second type of output pattern. And hence the total distinguishable discrete and uniformly-distributed refractive indices are $3\left(=2^{k+1}-1\right.$ for the case of $k=1)$.

Secondly, we select the case of $k=2$ as an example. Consider a two-level photonic digital sensor of which top view is shown in Figure 2 and its 3D structure is omitted for simplicity. There is one $\left(=2^{j-1}\right.$ for $j=1)$ directional coupler in the first level of the device and there are two $\left(=2^{j-1}\right.$ for $\left.j=2\right)$ directional couplers in the second level of the sensor. The total number of the directional couplers is three $\left(=2^{k}-1\right.$ for $k=2$ ). The authors still denote $L_{1,1}$ as the length of the closely-coupled section of the directional coupler in the first level. Let $B_{\mathrm{z}, 1}$ and $B_{\mathrm{x}, 1}$ separately denote the lateral and the longitudinal offsets of the each $S$-bend of the directional coupler in the first level. And let $B_{\mathrm{z}, 2}$ and $B_{\mathrm{x}, 2}$ be the lateral and the longitudinal offsets of the each $S$-bend in the second level of the sensor. The distance between two adjacent output ports of the photonic digital sensor is still denoted by $G$. The lengths of the directional couplers in the second level are respectively denoted as $L_{2,1}$ and $L_{2,2}$.

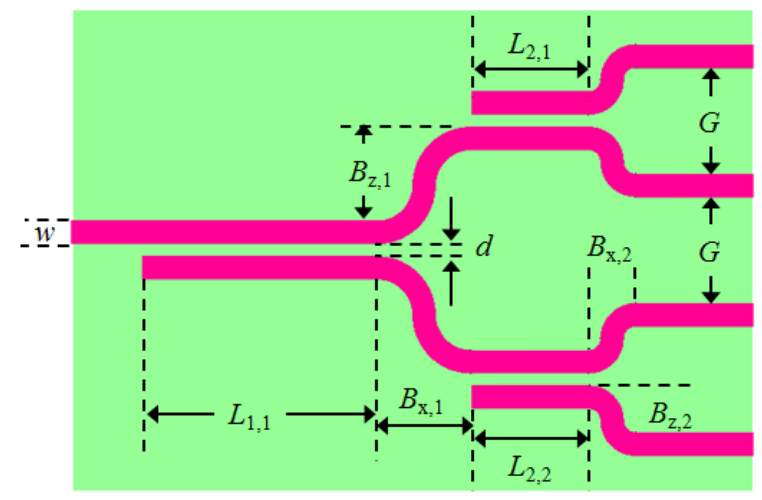

Fig. 2 The top view of two-level photonic digital sensor.

For $k=2$ case, adopting (2) and (3a), we select the lengths $L_{1,1}, L_{2,1}$, and $L_{2,2}$ as follows

$$
\begin{gathered}
L_{1,1}=\operatorname{ROUND}\left(\frac{L_{c, 3}}{\Delta L}\right) L_{c, 0} \\
\approx\left[\operatorname{ROUND}\left(\frac{L_{c, \mathrm{~s}}}{\Delta L}\right)+1\right] L_{\mathrm{c}, 1} \approx\left[\operatorname{ROUND}\left(\frac{L_{c, \mathrm{~s}}}{\Delta L}\right)+2\right] L_{\mathrm{c}, 2} \approx\left[\operatorname{ROUND}\left(\frac{L_{c, \mathrm{~s}}}{\Delta L}\right)+3\right] L_{\mathrm{c}, 3} \\
L_{2,1}=\operatorname{ROUND}\left(\frac{L_{c, 2}}{2 \Delta L}\right) L_{c, 0} \approx\left[\operatorname{ROUND}\left(\frac{L_{c, 2}}{2 \Delta L}\right)+1\right] L_{\mathrm{c}, 2}
\end{gathered}
$$

and

Brazilian Microwave and Optoelectronics Society-SBMO received 01 Nov 2017; for review 09 Nov 2017; accepted 03 March 2018 Brazilian Society of Electromagnetism-SBMag 


$$
L_{2,2}=\operatorname{ROUND}\left(\frac{L_{c, \mathrm{~s}}}{2 \Delta L}\right) L_{c, 1} \approx\left[\operatorname{ROUND}\left(\frac{L_{c, 2}}{2 \Delta L}\right)+1\right] L_{\mathrm{c}, 3}
$$

where the following approximations have been utilized in (5a)-(5c): $\operatorname{ROUND}\left(L_{\mathrm{c}, 3} / \Delta L\right) \approx L_{\mathrm{c}, 3} / \Delta L$, $\operatorname{ROUND}\left(L_{\mathrm{c}, 2} / \Delta L\right) \approx L_{\mathrm{c}, 2} / \Delta L, \operatorname{ROUND}\left(L_{\mathrm{c}, 3} / 2 \Delta L\right) \approx L_{\mathrm{c}, 3} / 2 \Delta L$, and $L_{\mathrm{c}, 3} / L_{\mathrm{c}, 1} \approx L_{\mathrm{c}, 3} / L_{\mathrm{c}, 2} \approx 1$ in case of $\Delta L<<L_{\mathrm{c}, 0}$. Notice that $L_{1,1}$ in (5a) for the case of $k=2$ is different from $L_{1,1}$ in (4) for the case of $k=1$. The numerator of the argument in $\operatorname{ROUND}(\cdot)$ of $(5 \mathrm{a})$ is $L_{\mathrm{c}, 3}$, which is the shortest one among the four coupling lengths: $\left(L_{\mathrm{c}, 0}, L_{\mathrm{c}, 1}, L_{\mathrm{c}, 2}, L_{\mathrm{c}, 3}\right)$. Moreover, the numerator of the argument in $\operatorname{ROUND}(\cdot)$ of $(5 \mathrm{~b})$, $L_{\mathrm{c}, 2}$, is the shorter one between the two coupling lengths: $\left(L_{\mathrm{c}, 0}, L_{\mathrm{c}, 2}\right)$. And the numerator in $(5 \mathrm{c}), L_{\mathrm{c}, 3}$, is the shorter one between another two coupling lengths: $\left(L_{\mathrm{c}, 1}, L_{\mathrm{c}, 3}\right)$. On the other hand, the denominator of the argument in $\operatorname{ROUND}(\cdot)$ of (5a) is $\Delta L$ but the denominators in (5b) and (5c) are $2 \Delta L$. Assume $\operatorname{ROUND}\left(L_{\mathrm{c}, 3} / \Delta L\right)$ is even, (5a) shows that that $L_{1,1}$ is approximately two sequential even multiple of $L_{\mathrm{c}, 0}$ and $L_{\mathrm{c}, 2}$. But $L_{1,1}$ is approximately two sequential odd multiples of $L_{\mathrm{c}, 1}$ and $L_{\mathrm{c}, 3}$. If the refractive index of the fluidic droplet is $n_{0}\left(=n_{\mathrm{cl}}\right)$ or $n_{2}\left(=n_{\mathrm{cl}}+2 \Delta n\right)$, the majority of the incident light will arrive at the upper output port of the directional coupler in the first level of the sensor in Figure 2. On the other hand, if the refractive index of the fluid droplet is $n_{1}\left(=n_{\mathrm{cl}}+\Delta n\right)$ or $n_{3}\left(=n_{\mathrm{cl}}+3 \Delta n\right)$, the major portion of the optical power will be finally coupled to the lower output port of the directional coupler in the first level. Conversely, if $\operatorname{ROUND}\left(L_{\mathrm{c}, 3} / \Delta L\right)$ is odd, all the above output results are exchanged. Therefore, the directional coupler in the first level of the sensor can classify the refractive indices of the 4 kinds of fluid samples $\left(n_{0}, n_{1}, n_{2}, n_{3}\right)$ into two smaller groups: $\left(n_{0}, n_{2}\right)$ and $\left(n_{1}, n_{3}\right)$. Similarly, we also can show that one of the directional couplers in the second level is capable of distinguishing $n_{0}$ and $n_{2}$, and the other one is capable of telling $n_{1}$ from $n_{3}$. Assume the refractive index of the fluid droplet is $n_{0}$. If $\operatorname{ROUND}\left(L_{\mathrm{c}, 3} / 2 \Delta L\right)$ is even, $L_{2,1}$ in $(5 \mathrm{~b})$ is an even multiple of $L_{\mathrm{c}, 0}$ but it is an approximate odd multiple of $L_{\mathrm{c}, 2}$. In this case, the major optical power will reach the upper output port of the upper directional coupler in the second level of the sensor in Figure 2. Conversely, if the refractive index of the fluid droplet is $n_{2}$, the majority of the incident light will arrive at the lower output port of the same directional coupler. Alternately, if $\operatorname{ROUND}\left(L_{\mathrm{c}, 3} / 2 \Delta L\right)$ is odd, the previously-mentioned output results of the upper directional coupler in the second level of the sensor should be exchanged. And hence we can distinguish $n_{0}$ and $n_{2}$. The similar description can explain the lower directional coupler in the second level of the sensor can tell $n_{1}$ from $n_{3}$. Regardless of either case in the above descriptions, there is only one output port, corresponding to the respective one of $n_{0}, n_{1}, n_{2}$, and $n_{3}$, capable of radiating the strongest light from the two-level photonic digital sensor for the first type of output patterns. On the other hand, the following computer simulation results show that we can determine $n_{0.5}, n_{1.5}$, and $n_{2.5}$ by observing which two output ports radiate the same bright lights for the second type of output patterns. And thus the total distinguishable discrete and uniformly-distributed refractive indices of fluidic samples are $7\left(=2^{k+1}-1\right.$ for the case of $\left.k=2\right)$.

In the next paragraph, the authors still select the one-level and the two-level optical digital sensors 
as the examples for numerical simulation to demonstrate the validity of our design. Although we give the case of $k \leq 2$ as examples in this paper, the similar design rule can be expanded to the other case of arbitrary integer $k \geq 3$. And it is easily shown that the $k$-level photonic digital sensor contains $2^{j-1}$ directional couplers in the $j_{\text {th }}$ level for $1 \leq j \leq k$ by induction method.

\section{NUMERICAL SIMULATION RESULTS}

The optical wavelength is $0.633 \mu \mathrm{m}$ in our simulation. And the following typical structural parameters are given in this work: $w=0.6 \mu m, t=0.7 \mu m, d=0.3 \mu m, n_{\mathrm{g}}=1.59, n_{\mathrm{s}}=1.46, n_{\mathrm{cl}}=1.33$, and $n_{\mathrm{ch}}=1.34$, respectively. The above wavelength and the structural parameters are as same as given in $[6$, 11]. In this work, all the BPM simulation results are obtained by executing Rsoft-BeamPROP software. For the case of single-level photonic digital sensor $(k=1)$, the values of $B_{\mathrm{x}, 1}, B_{\mathrm{z}, 1}$, and $G$ are determined by BPM simulation such that the relative optical power loss in each bending section is less than $1 \%$ and no mutual power coupling occurs between two adjacent output ports. Afterwards we obtain $B_{\mathrm{x}, 1}=0.6 \mu \mathrm{m}, B_{\mathrm{z}, 1}=30 \mu \mathrm{m}$, and $G=1.5 \mu \mathrm{m}$. For designing the other structural parameters of the one-level photonic digital sensor; firstly, we utilize BPM simulation to obtain the coupling lengths: $L_{\mathrm{c}, 0}=58.8 \mu m$ and $L_{\mathrm{c}, 1}=56.4 \mu m$, which are respectively corresponding to the cases of $n_{0}$ and $n_{1}$. Secondly, the directional coupler length $L_{1,1}$ within the one-level photonic digital sensor can be determined by (4). And then we obtain $L_{1,1}=1411 \mu \mathrm{m}$ for the case of $k=1$.
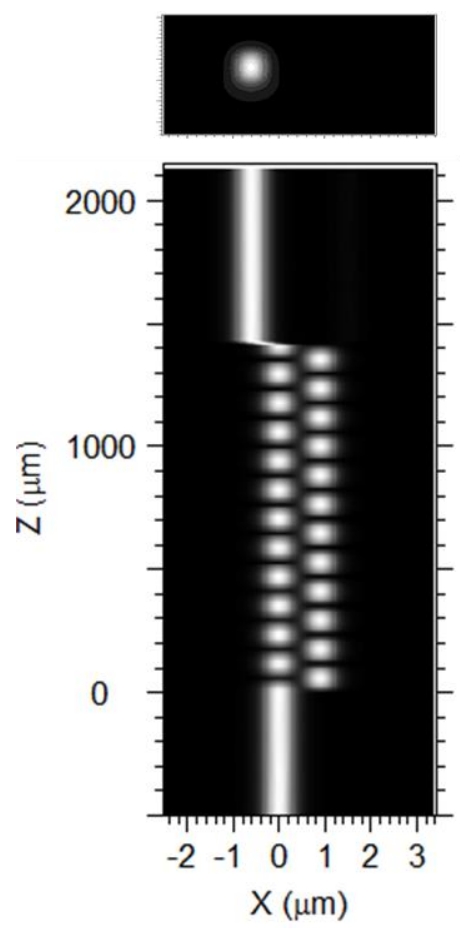

(a)
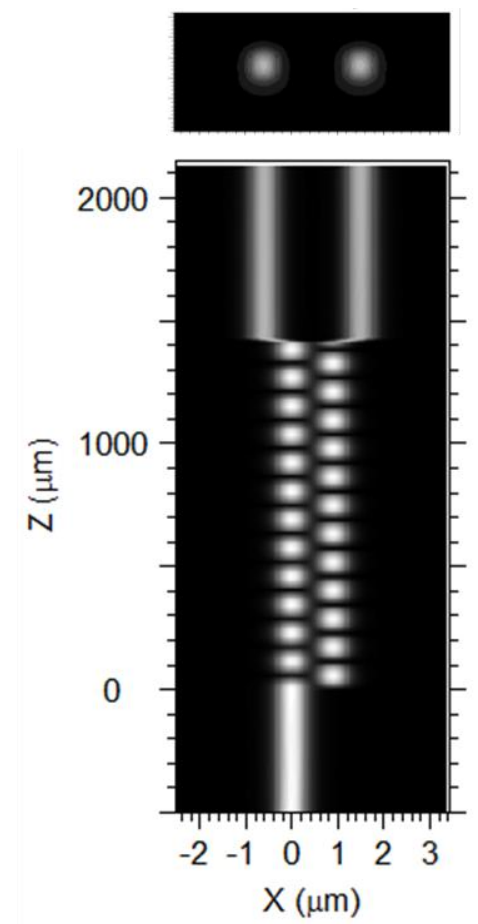

(b)
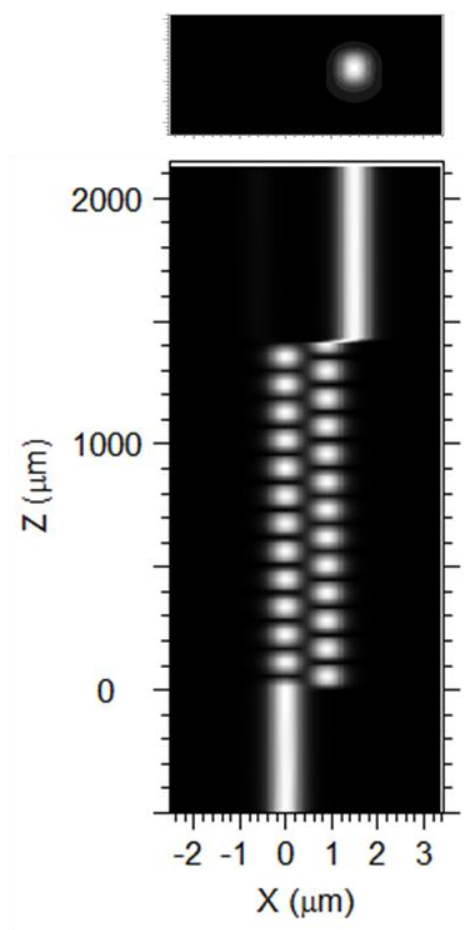

(c)

Fig. 3 The different propagation paths of the incident light within the two-level all-optical digital sensor in case of (a) $n_{\mathrm{c}}=n_{0}$, (b) $n_{\mathrm{c}}=n_{0.5}$, and (c) $n_{\mathrm{c}}=n_{1}$. 
Figures 3(a)-(c) show the BPM simulation results of depositing $3\left(=2^{k+1}-1\right.$ for $\left.k=1\right)$ species of distinct fluidic samples, whose refractive indices are expressed by $n_{0}, n_{0.5}$, and $n_{1}$, respectively. Among them, the upper part of each sub-figure is the optical power distribution on end face of the output ports (the front view). And the lower part of each sub-figure is the propagating optical power distribution along the directional coupler of the one-level photonic digital sensor (the top view). It is found that the propagating light is continuously coupled to and fro between the initially-incident waveguide and its adjacent one of the directional coupler in case the refractive index of the fluid is $n_{0}\left(=1.33=n_{\mathrm{cl}}\right)$. At final, the major optical power appears at the left output port of the one-level photonic digital sensor, as shown in Figure 3(a). If the refractive index of the fluidic sample increases to $n_{0.5}\left(=n_{0}+0.5 \Delta n\right)$, Figure 3(b) describes that the two output ports radiate the same optical power. Increasing the refractive index of the fluidic sample to $n_{1}\left(=n_{\mathrm{ch}}=n_{0}+\Delta n\right)$, Figure 3(c) presents that the major optical power reaches the right output port. Figures 3(a) and 3(c) are both the first type of output patterns because only one output port can emit bright light, but Figure 3(c) presents the second type of output pattern because the two output ports radiate the identical output optical power. The above sub-figures show that two $\left(=2^{k}\right.$ for $\left.k=1\right)$ of them are of the first type of output patterns, and one $\left(=2^{\mathrm{k}}-1\right.$ for $\left.k=1\right)$ of them is of the second type of output pattern.

For designing the two-level photonic digital sensor $(k=2)$, we still utilize BPM simulation to obtain $B_{\mathrm{x}, 1}=1.2 \mu m, B_{\mathrm{z}, 1}=60 \mu m, B_{\mathrm{x}, 2}=0.6 \mu m, B_{\mathrm{z}, 2}=30 \mu m$, and $G=1.5 \mu m$ for avoiding mutual coupling between paralell waveguides. And the BPM simulation results show that the coupling lengths are $L_{\mathrm{c}, 0}=58.8 \mu m$, $L_{\mathrm{c}, 1}=58.0 \mu \mathrm{m}, L_{\mathrm{c}, 2}=57.2 \mu \mathrm{m}$, and $L_{\mathrm{c}, 3}=56.4 \mu \mathrm{m}$, which are corresponding to the cases of $n_{0}, n_{1}, n_{2}$, and $n_{3}$, respectively. The directional couplers lengths within the two-level photonic digital sensor: $L_{1,1}=4116 \mu m, L_{2,1}=2058 \mu m$, and $L_{2,2}=2030 \mu m$, can be separately determined by (5a)-(5c).

Figures $4(\mathrm{a})-(\mathrm{g})$ show the BPM simulation results of depositing $7\left(=2^{k+1}-1\right.$ for $\left.k=2\right)$ species of distinct fluidic samples, whose refractive indices are respectively $n_{0}, n_{0.5}, n_{1}, n_{1.5}, n_{2}, n_{2.5}$, and $n_{3}$ on the surface of the two-level photonic digital sensor with the above structural parameters. Among them, Figures 4(a), 4(c), 4(e), and 4(g) present the first type of output patterns. Only one of the four output ports of the sensor, which are respectively corresponding to the $4\left(=2^{k}\right.$ for $\left.k=2\right)$ cases of $n_{0}, n_{1}, n_{2}$, and $n_{3}$, can have the strongest light. The authors label ordinal numbers to the output ports from the left to the right. Figure 4(a) shows that the propagating optical wave is continuously coupled back and forth between the initially-incident waveguide and its adjacent one of the directional coupler in the first level of the sensor if the refractive index of the fluid is $n_{0}\left(=1.33=n_{\mathrm{cl}}\right)$. Afterwards the major optical power enters the left directional coupler in the second level of the structure, and then the propagating light sustains coupling to and fro between the both waveguides. Finally, the maximal optical power appears at the $1^{\text {st }}$ output port of the two-level photonic digital sensor, as shown in Figure 4(a). Increasing the refractive index to $n_{1}\left(=n_{0}+\Delta n\right)$, Figure 4(c) presents that the strongest light reaches the $4^{\text {th }}$ output port. In case the refractive index of the fluidic sample becomes $n_{2}\left(=n_{0}+2 \Delta n\right)$, Figure $4(\mathrm{e})$ describes that the $2^{\text {nd }}$ output port has the maximal optical power. If the refractive index of the fluid is Brazilian Microwave and Optoelectronics Society-SBMO received 01 Nov 2017; for review 09 Nov 2017 ; accepted 03 March 2018 
$n_{3}\left(=n_{0}+3 \Delta n=1.34=n_{\mathrm{ch}}\right)$, Figure $4(\mathrm{~g})$ demonstrates that the strongest light arrives at the $3^{\text {rd }}$ output port. On the other hand, Figures 4(b), 4(d), and 4(f) present the second type of output patterns. Three specific pairs of output ports, which are respectively corresponding to the $3\left(=2^{k}-1\right.$ for $\left.k=2\right)$ cases of $n_{0.5}, n_{1.5}$, and $n_{2.5}$, can have the same brightest lights. In case the refractive index of the fluid is $n_{0.5}\left(=n_{0}+0.5 \Delta n\right)$, Figure $4(\mathrm{~b})$ shows that $1^{\text {st }}$ and the $4^{\text {th }}$ output ports of the two-level all-optical digital sensor have the same maximal optical power. Increasing the refractive index to $n_{1.5}\left(=n_{0}+1.5 \Delta n\right)$, Figure 4(d) presents that the both strongest lights appear at the $2^{\text {nd }}$ and $4^{\text {th }}$ output ports. If the refractive index of the fluidic sample becomes $n_{2.5}\left(=n_{0}+2.5 \Delta n\right)$, Figure 4(f) describes that the $2^{\text {nd }}$ and the $3^{\text {rd }}$ output ports have the same brightest lights.

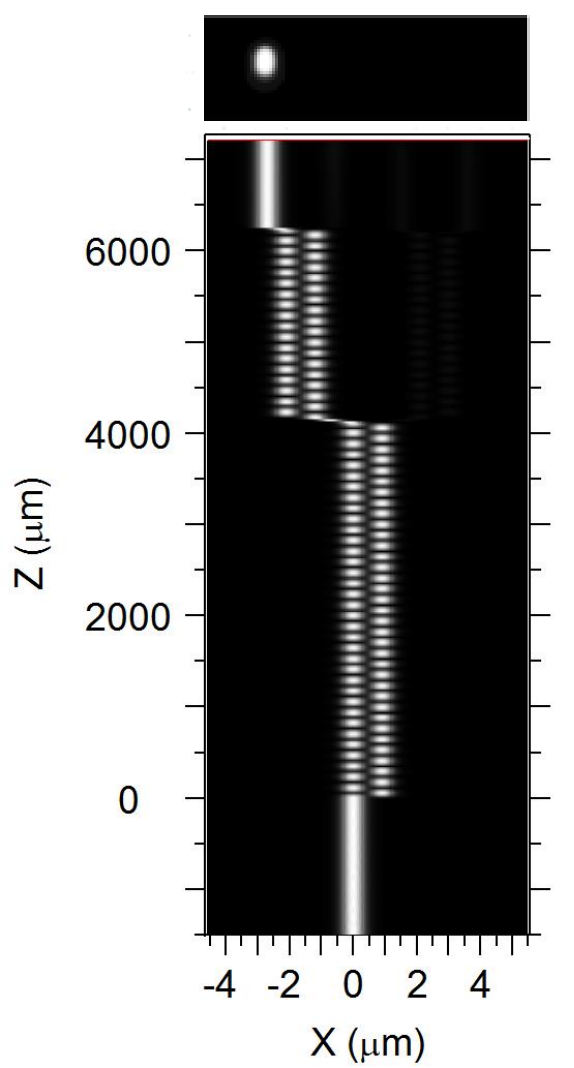

(a)

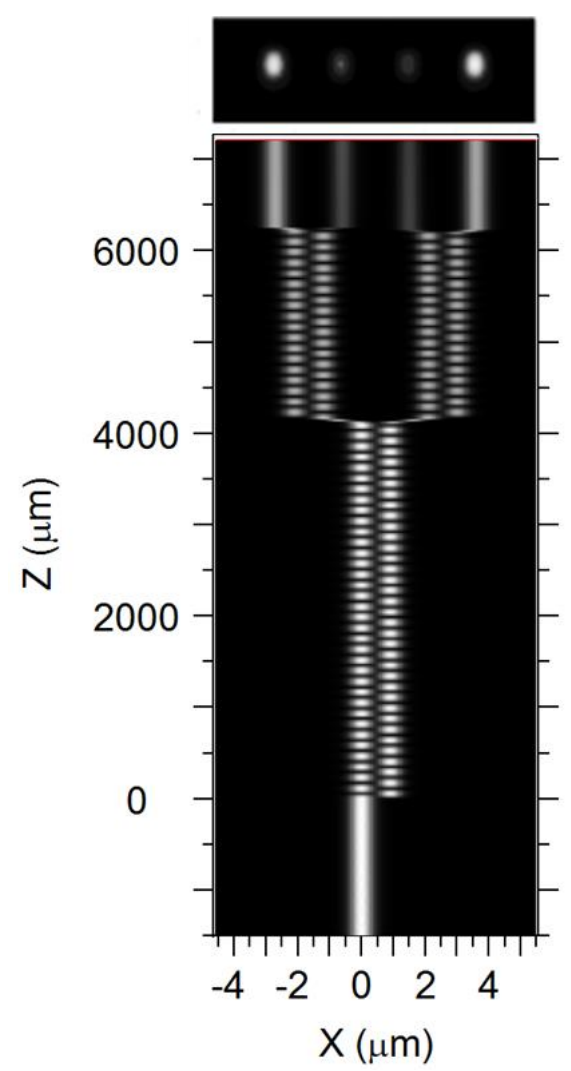

(b) 


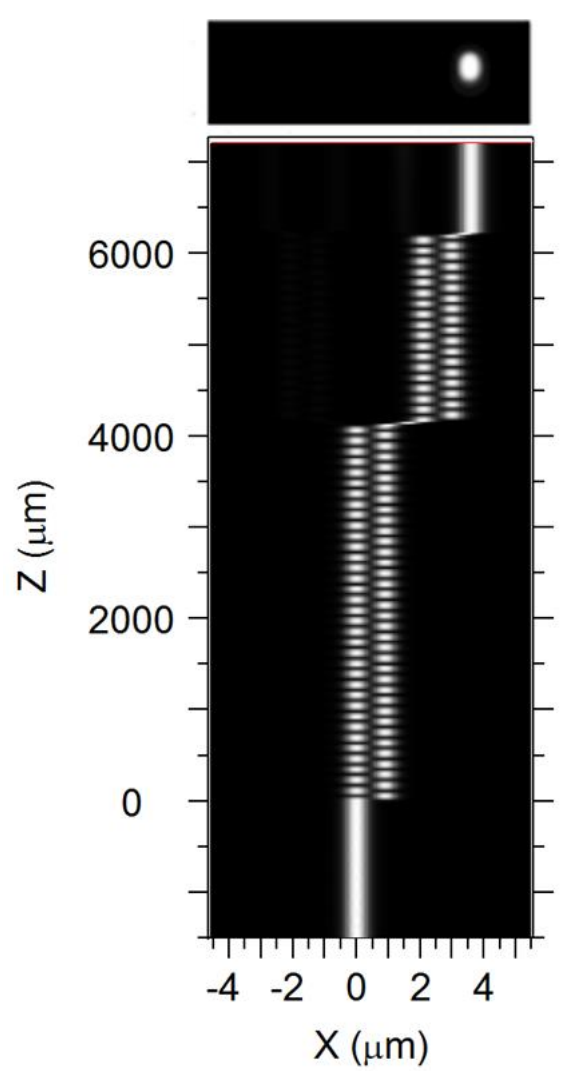

(c)

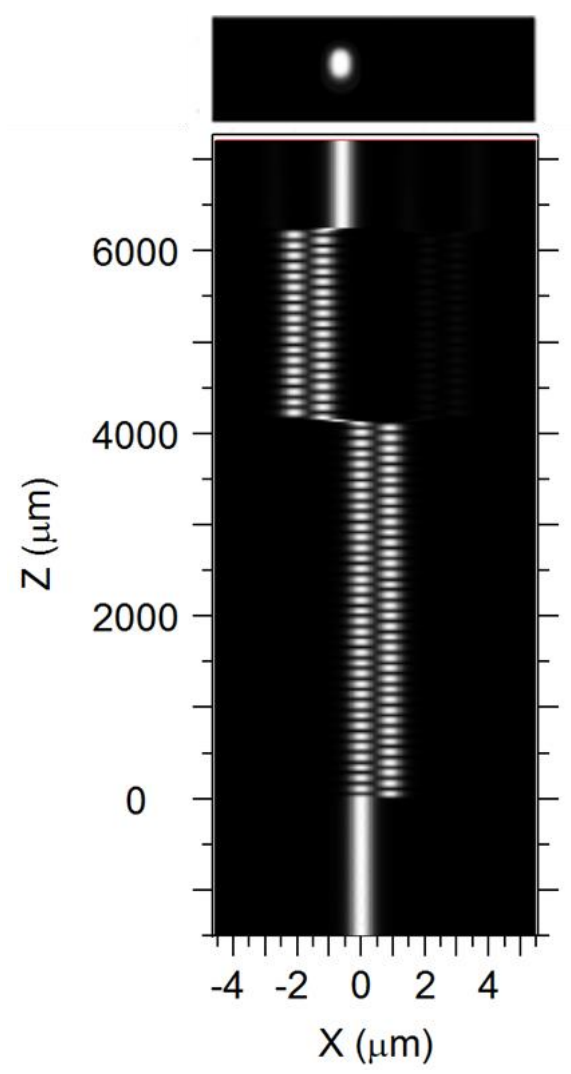

(e)

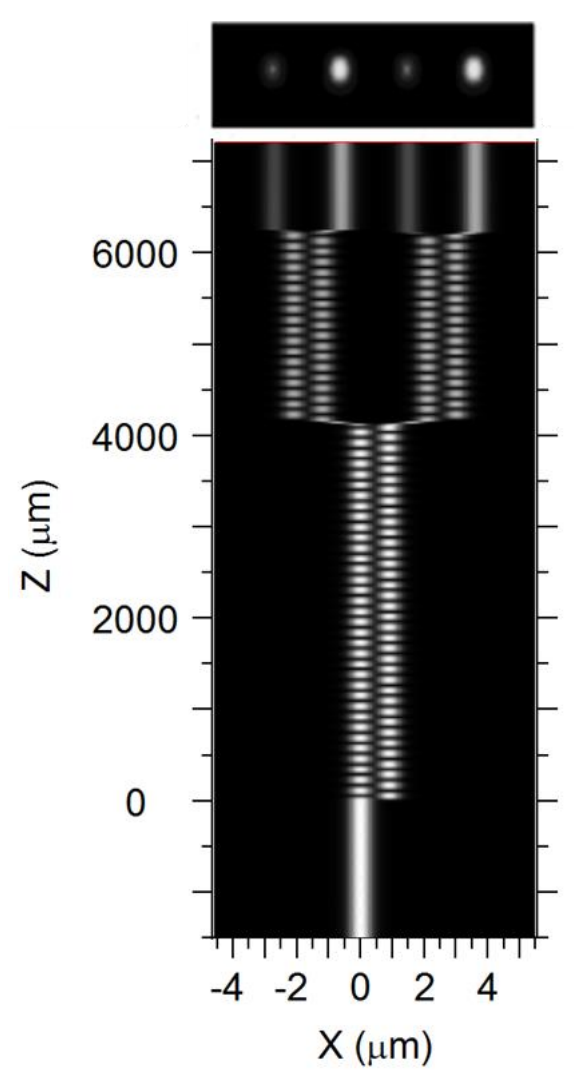

(d)

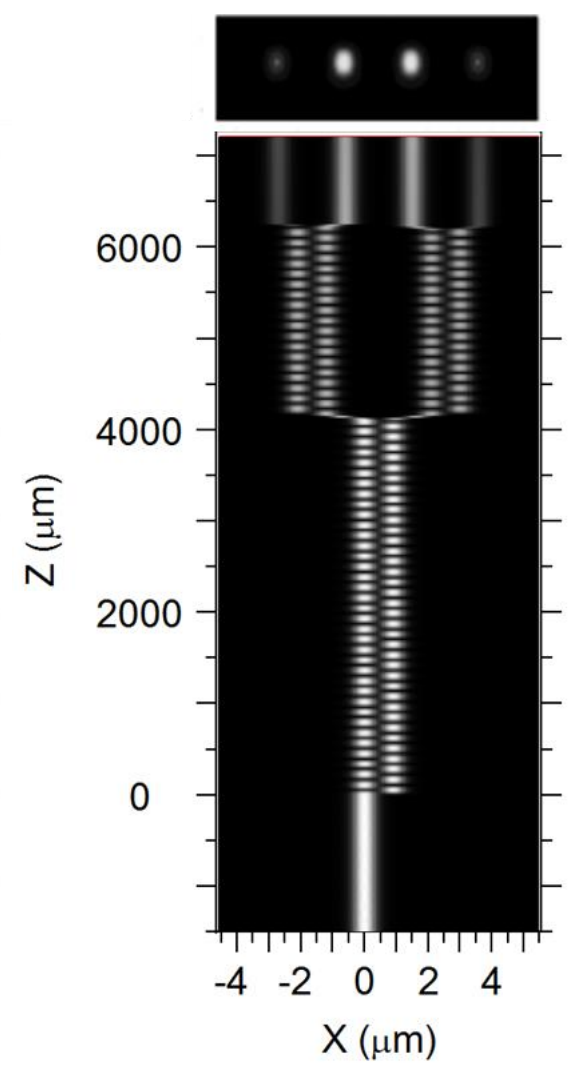

(f) 


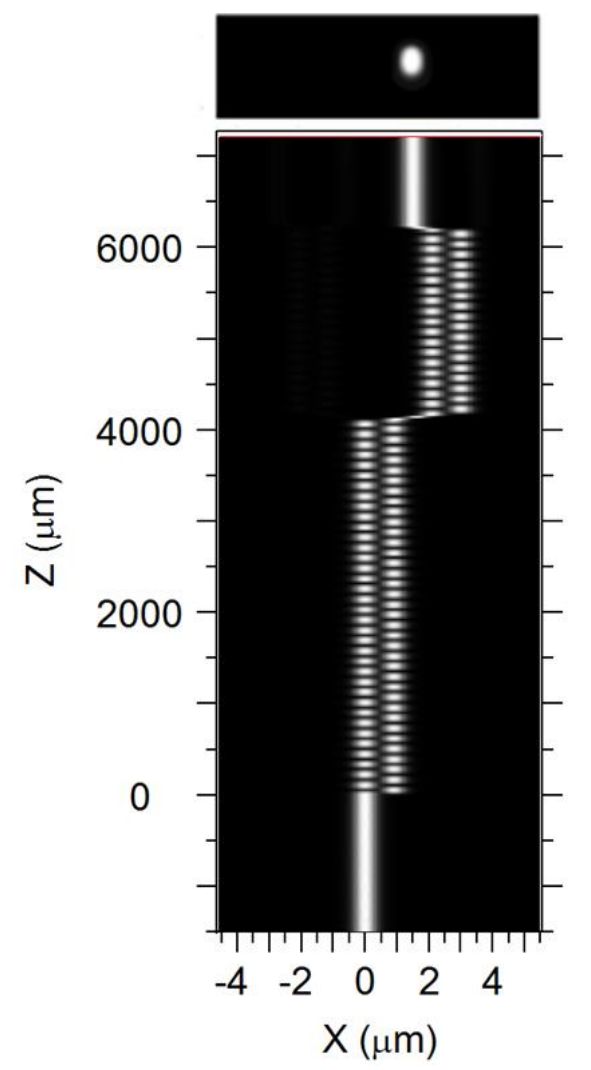

(g)

Fig. 4 The different propagation paths of the incident light within the two-level all-optical digital sensor in case of (a) $n_{\mathrm{c}}=n_{0}$, (b) $n_{\mathrm{c}}=n_{0.5}$, (c) $n_{\mathrm{c}}=n_{1}$, (d) $n_{\mathrm{c}}=n_{1.5}$, (e) $n_{\mathrm{c}}=n_{2}$, (f) $n_{\mathrm{c}}=n_{2.5}$, and (g) $n_{\mathrm{c}}=n_{3}$.

Similarly, we still can simulate the performance of the $k$-level $(k \geqq 3)$ photonic digital sensor, which is capable of distinguishing $15\left(=2^{k+1}-1\right.$ for $\left.k=3\right), 31\left(=2^{k+1}-1\right.$ for $\left.k=4\right), 63\left(=2^{k+1}-1\right.$ for $\left.k=5\right), \ldots$, and more species of fluidic samples with uniformly-distributed refractive indices. The authors omit them in this paper for simplicity.

As previous mention, a simple and high-sensitivity refractive sensor is presented. With our proposed digital sensor, the users can observe the output optical spots and determine the refractive index directly. Additional apparatus such as power voltmeter or broadband light source are not required during the process of the measurement.

\section{CONCLUSIONS}

From our proposed design principle and simulation results, some conclusions are drawn as follows. Our proposed $k$-level photonic digital sensor based on directional couplers with specific lengths is capable of determining $\left(2^{k+1}-1\right)$ species of fluidic samples with uniformly-distributed refractive indices. Without the necessity of the precisely measuring the optical power distributions/ratios at the output ports, we just need to observe or judge which output port radiates the maximal optical power, and then the corresponding refractive index of the fluidic sample is obtained. Therefore, $2^{k}$ species of 
fluidic samples can be distinguished by observing the first type of output patterns. For the second type of output patterns, it is just necessary to observe and judge which pairs of output ports emit the same strongest lights. And then the extra $\left(2^{k}-1\right)$ refractive indices of the tested fluidic samples can be obtained. Although only the numerical simulation results of $k=1$ and 2 are presented and validate our design in this work, the design principle and the corresponding performances of the $k$-level photonic digital sensor can be easily expanded to the other case of arbitrary integer $k \geq 3$.

\section{REFERENCES}

[1] A. C. Walker, "Application of bistable optical logic gate arrays to all-optical digital parallel processing", Appl. Opt., vol. 25, pp. 1578-1585,1986.

[2] R. Slavík, F. Parmigiani, J. Kakande, C. Lundström, M. Sjödin, P. A. Andrekson, R. Weerasuriya, S. Sygletos, A. D. Ellis, L. Grüner-Nielsen, D. Jakobsen, S. Herstrøm, R. Phelan, J. O'Gorman, A. Bogris, D. Syvridis, S. Dasgupta, P. Petropoulos and D. J. Richardson, "All-optical phase and amplitude regenerator for next-generation telecommunications systems", Nature Photon., vol. 4, pp. 690-695, 2010.

[3] G. Contestabile, "A Multirate All-Optical Aggregator for Digital Back-Haul and PON Uplink", IEEE Photon. Technol. Lett., vol. 26, pp. 862-865, 2016.

[4] T. Takeo and H. Hattori, "Optical Fiber Sensor for Measuring Refractive Index", Japanese J. of Appl. Physics, vol. 21, pp. 1509-1512, 1982.

[5] H. H. Zhu et al., "High-sensitivity optical sensors based on cascaded reflective MZIs and microring resonators", Opt. Express, vol. 25, pp. 28612-28618, 2017.

[6] B. J. Luff, R. D. Harris, J. S. Wilkinson, R. Wilson, and D. J. Schiffrin, "Integrated-optical directional coupler biosensor," Opt. Lett., vol. 21, pp. 618-620, 1996.

[7] Lin Luan, Randall D. Evans, Nan M. Jokerst, Richard B. Fair, "Integrated optical sensor in a digital microfluidic platform", IEEE Sensors J., vol. 8, pp. 628-635, 2008.

[8] H. Mohana, Gopalb, A. Kumarc, "Design and analyze the refractive index sensor having the discontinuity between sensing region and cladding area by large core diameter", Procedia Technol., vol. 17, pp. 289-294, 2014.

[9] J. L. Lim, D. J. J. Hu, P. P. Shum, and Y. Wang, "Design and Analysis of Microfluidic Optical Fiber Device for Refractive Index Sensing", IEEE Photon. Technol. Lett., vol. 26, pp. 2130-2133, 2014.

[10] K. Uchiyamada, K. Okubo, M. Yokokawa, E. T. Carlen, K. Asakawa, and H. Suzuki,"Micron scale directional coupler as a transducer for biochemical sensing”, Opt. Express, vol. 23, pp. 17156-17168, 2015.

[11] R.-C. Lu and K.-Y. Lee, "Sensing refractive indices of fluids by wavelength-tunable laser and novel multi-stage directional couplers", J. Optical Communications, vol. 38, pp. 147-151, 2017.

[12] A. Yariv, "Coupled-mode theory for guided-wave optics," IEEE J. Quantum Electron., vol. 9, pp. 919-933, 1973. 